\title{
Perspective
}

\section{Extremely low vaccine effectiveness against influenza H3N2 in the elderly during the 2012/2013 flu season}

\author{
David J. Kelvin ${ }^{1,2,3}$, Amber Farooqui ${ }^{1,2}$ \\ ${ }^{1}$ ShantouUniversity Medical College, 22 Xingling Road, Shantou, GD 515041, People's Republic of China \\ ${ }^{2}$ The Journal of Infection in Developing Countries \\ ${ }^{3}$ Division of Experimental Therapeutics, Toronto General Research Institute, University Health Network, 101 \\ College Street, Toronto, Ontario M5G1L7, Canada
}

Key words: influenza; vaccines; elderly; H3N2

J Infect Dev Ctries 2013; 7(3):299-301.

(Received and Accepted 13 March 2013)

Copyright $(\subset) 2013$ Kelvin and Farooqui. This is an open-access article distributed under the Creative Commons Attribution License, which permits unrestricted use, distribution, and reproduction in any medium, provided the original work is properly cited.

\section{Curious anomalies in vaccine effectiveness during the 2012/2013 flu season}

The 2012/2013 influenza season is winding down now in North America, Europe and other Northern hemisphere locations, and interim estimates for vaccine effectiveness are now available from multiple sources $[1,2,3,4]$. The most striking statistic reported $[3,4]$ is that the vaccine displayed extremely low effectiveness against A/H3N2 in the elderly. This data has significant implications for future vaccine design, influenza surveillance, and treatment of the elderly during influenza outbreaks and epidemics.

In the early months of each year, the Centers for Disease Control in Atlanta and the World Health Organization make recommendations for the vaccine strains of influenza to be used by vaccine producers for manufacturing influenza vaccines [5] in the northern hemisphere for use in the following influenza season. In the absence of a pandemic, usually three strains of influenza are chosen for vaccine production: two influenza A viruses, one from the H1N1 subtype and one from the H3N2 subtype, and one B influenza virus. In early 2012 the three chosen viruses for the 2012/2013 flu season were A/California/7/2009 (H1N1)pdm09-like virus, A/Victoria/361/2011 (H3N2)-like virus, and B/Wisconsin/1/2010-like virus [5].

The 2012/2013 influenza season was early and moderately severe in the United States [6]. The predominant circulating strains were antigenically similar to A/Victoria/2011-like (H3N2) and $\mathrm{B} /$ Wisconsin/1/2010-like $(69 \%$ of the $\mathrm{B}$ viruses detected, Yamagata lineage) and B/Victoria (31\% of the $\mathrm{B}$ viruses detected, Victoria lineage). The early flu season allowed for interim vaccine effectiveness casecontrol studies to be determined for Canada [1], the United Kingdom [2], Denmark [3] and the United States [4]. The studies from Canada, the United States, and the United Kingdom have similar overall vaccine effectiveness for all influenza strains across the entire population (see Table 1). The effectiveness ranged from $38 \%$ to $49 \%$ for A viruses and $46 \%$ to $50 \%$ for all influenza viruses. B virus effectiveness was $52 \%$ and $67 \%$ respectively for the United Kingdom and the United States.

These numbers for the general population are not so surprising and appear on the surface to be in line with what has been reported for previous years. The disturbing statistic is evident when effectiveness is broken down for age (Table 2). The studies from the United States [4] and Denmark [3] both show that vaccine effectiveness in the elderly ( $\geq 65$ years of age) is extremely low (see last row of Table 2). What is even more perplexing is that the younger age groups in the American study show between $46 \%$ and $58 \%$ vaccine effectiveness against $\mathrm{H} 3 \mathrm{~N} 2$, validating that the vaccine can elicit protective responses, at least in people younger than 65 years of age. Furthermore, the elderly group in both the American study and the Danish studies show that vaccine effectiveness against B viruses was relatively high at $69 \%$ and $67 \%$ for the two countries (Table 2). 
Table 1.Vaccine effectiveness against A and B influenza viruses

\begin{tabular}{|l|l|l|l|}
\hline Country & $\begin{array}{l}\text { H3N2 (VE) } \\
\text { or A viruses }\end{array}$ & All influenza (VE) & B virus (VE) \\
\hline Canada (ref 1) & $38 \%$ (H3N2) & $46 \%$ & NA \\
\hline United States (ref 4) & $47 \%$ (H3N2) & $56 \%$ & $67 \%$ \\
\hline United Kingdom (ref 2) & $49 \%$ (A viruses) & $51 \%$ & $52 \%$ \\
\hline
\end{tabular}

VE - Vaccine effectiveness; NA - Not available

Table 2.Age distribution of vaccine effectiveness 2012-2013

\begin{tabular}{|l|l|l|l|l|}
\hline & \multicolumn{2}{|l|}{ H3N2 Vaccine Effectiveness } & \multicolumn{2}{l|}{ B virus vaccine effectiveness } \\
\hline Age Group & Denmark * & United States** & Denmark* & United States** \\
\hline 6 months -17yrs & NA & $58 \%$ & NA & $64 \%$ \\
\hline $18-49$ & NA & $46 \%$ & NA & $68 \%$ \\
\hline $50-64$ & NA & $50 \%$ & NA & $75 \%$ \\
\hline$\geq 65$ & $-11 \%$ & $9 \%$ & $69 \%$ & $67 \%$ \\
\hline
\end{tabular}

*Reference $-3 ; * *$ Reference $-4 ; \mathrm{NA}=$ Not available

\section{Why is the H3N2 vaccine effectiveness so low in the elderly for the $2012 / 2013$ season?}

Part of the answer to this question may reside in antigenic changes identified in the HA1 gene of the H3N2 viruses that circulated in Canada and Denmark $[1,3]$. Skowronski and colleagues [1] and Bragstad and colleagues[3] both identified numerous mutations in the antigenic regions of the HA gene, thereby constituting a possible antigenic drift of the A/Victoria/361/2011 (H3N2)-like virus to the present circulating H3N2 strains in North America and Europe. Antigenic studies will provide insight into this possibility.

Interestingly, the antigenic shift does not explain everything; the vaccine was still capable of eliciting effective immune responses against $\mathrm{H} 3 \mathrm{~N} 2$ in the young. A simple interpretation of this data is that the elderly generate a narrow antibody response to the vaccine strain of $\mathrm{H} 3 \mathrm{~N} 2$ which is not capable of protecting against a $\mathrm{H} 3 \mathrm{~N} 2$ virus with shifted antigenicity. Moreover, younger vaccinated people likely generate an immune response with greater breadth which can protect against shifted viruses. If this is so, how can vaccine manufacturers create a vaccine that brings out a larger breadth of antibody responses? Many adjuvants currently await testing, but we must learn more about the immune system in the elderly to design more effective vaccines. We must avoid a random sampling of adjuvants in the hope one will work. We need good rational design based upon fundamental studies in the elderly.

These questions are important and are farreaching. The elderly are a high-risk population due to their lack of robust immune responses and many vaccines are in the pipeline for clinical studies. Fundamental studies will benefit vaccine development for West Nile Virus, RSV, and many other infectious agents.

On the otherhand, it is important to maintain vigilant real-time surveillance for shifting influenza viruses. Antigenic characterization of these viruses will aid in preparing front-line health-care workers with treatment options that include anti-viral drugs for the elderly during flu season.

\section{Acknowledgements}

We would like to thank Professor Giovanni Fadda for the many years of friendship and mentorship. $\mathrm{He}$ is an extraordinary scientist, friend and humanist.

\section{References}

1. Skowronski D, Janjua N, De Serres G, Dickinson J, Winter A, Mahmud S, Sabaiduc S, Gubbay J, Charest H, Petric M, Fonseca K, Van Caeseele P, Kwindt T, Krajden M, Eshaghi A, Li Y (2013) Interim estimates of influenza vaccine effectiveness in 2012/13 from Canada's sentinel surveillance network, January 2013. Euro Surveill 18: 1-10. doi:pii: 20394.

2. McMenamin J, Andrews N, Robertson C, Fleming D, Durnall $\mathrm{H}$, von Wissmann B, Ellis J, Lackenby A, Cottrell S, Smyth B, Zambon M, Moore C, Watson J, Pebody R (2013)Effectiveness of seasonal 2012/13 vaccine in preventing laboratory-confirmed influenza infection in primary care in the United Kingdom - mid-season analysis 2012/13. Euro Surveill 18: 1-5. doi:pii: 20393.

3. Bragstad K, Emborg H, Kolsen Fischer T, Voldstedlund M, Gubbels S, Andersen B, Molbak K, Krause T (2013) Low vaccine effectiveness against influenza $\mathrm{A}(\mathrm{H} 3 \mathrm{~N} 2)$ virus among elderly people in Denmark in 2012/13: a rapid 
epidemiological and virological assessment. Euro Surveill 18: 11-17. doi:pii: 20397.

4. CDC (2013) Interim Adjusted Estimates of Seasonal Influenza Vaccine Effectiveness - United States, February 2013.MMWR 62: 119-123.

5. World Health Organization (WHO) (2012) Recommended composition of influenza virus vaccines for use in the 20122013 northern hemisphere influenza season. 23 February 2012 Available:

http://www.who.int/influenza/vaccines/virus/recommendation s/2012_13_north/en/index.html.[Accessed 13 March 2013].

6. CDC(2013)Fluview 2012-2013 influenza season. Atlanta, GA: US Department of Health and Human Services, CDC. Available: http://www.cdc.gov/flu/weekly/fluviewinteractive.htm [Accessed 13 March 2013].

\section{Corresponding author}

David J. Kelvin

Shantou University Medical College

22 Xinling Road

Shantou 515041, Guangdong, China

Telephone: +8675488573991

Fax: +8675488573991

Email: dkelvin@jidc.org

Conflict of interests: No conflict of interests is declared. 